\title{
A Mixed Variable Speed Reaching Law of Sliding Mode Control for Spacecraft Tracking System
}

\author{
Yao Zhang and Yu-Xin Zhao \\ College of Automation, Harbin Engineering University, Harbin 150001, China \\ Correspondence should be addressed to Yu-Xin Zhao; zhaoyuxin@hrbeu.edu.cn
}

Received 13 June 2014; Accepted 3 August 2014

Academic Editor: Ligang Wu

Copyright ( 2015 Y. Zhang and Y.-X. Zhao. This is an open access article distributed under the Creative Commons Attribution License, which permits unrestricted use, distribution, and reproduction in any medium, provided the original work is properly cited.

For spacecraft tracking control system, the reaching law election and controller design are two crucial and important problems. In this paper, spacecraft tracking system is considered as a discrete-time system, a mixed variable speed reaching law of SMC, and a controller for spacecraft tracking system has been investigated. Theory proves that this method can ensure the stability of spacecraft system and eliminate the chattering phenomenon. Furthermore, when spacecraft is inflicted by a certain external interference, the regulating function of neural network can ensure strong robustness of the system. Simulation results show that, compared with exponential reaching law and classical variable speed reaching law, the proposed reaching law has better suppress chattering effect and dynamic performance.

\section{Introduction}

Sliding mode variable structure control theory is essentially a kind of special nonlinear control, and its nonlinear performance is the discontinuity for the control [1]. Due to the rapid development of computer technology, discrete-time system is widely used in the actual spacecraft attitude control; therefore, the sliding mode control for spacecraft tracking system in the form of discrete-time is especially important $[1,2]$. In all kinds of nonlinear systems, chattering is caused by the influence of time delay and inertia in time or space, which affects the accuracy of control system. It may also stimulate strong oscillation of the unmodeled part and is harmful to the system [2]. Chattering is a fatal flaw of classical variable structure control system, which drives the high frequency part without modeling of system and increases the burden of the controller. Therefore, the suppression of chattering became an important subject in the field of sliding mode variable structure control.

In recent years, the sliding mode control method based on reaching law has become the focus of attention. Based on the reaching law, Gao et al. proposed the discrete sliding mode variable structure control [3]. Koshkouei and Zinober proposed the existence conditions of a new sliding mode and designed a new sliding mode control law [4]. But it is difficult to guarantee the robustness of sliding mode control effectively. Chen studied sliding mode control for the multiple input-output discrete-time system with disturbances and unknown parameters, and an adaptive reaching law was implemented to estimate the unknown term [5]. For the deterministic system, Bartoszewicz derived a quasisliding mode controller for discrete-time system [6], which can reduce chattering caused by high frequency. Zaidi et al. designed a proportional sliding mode control law for singlephase induction motor, and it has good convergence; however, there exists chattering phenomenon in waveform [7]. Niu et al. improved a sliding mode controller via reaching law, which is a kind of exponential reaching law and cannot reach zero in finite time [8]. Veselic et al. proposed a variable speed reaching law, which has high convergence speed in reaching stage; however, it has low speed in sliding motion stage [9]. Therefore, it is necessary to find a kind of reaching law, which can eliminate the chattering phenomenon.

In order to solve the chattering problem, many researches have been provided by using advanced control methods. Wu 
et al. proposed a sliding mode control with bounded L2 gain performance of Markovian jump singular systems, which is proved useful for time-delay system [10]. Soltanpour et al. designed a kind of fuzzy sliding mode control for nonlinear system, which can solve the chattering problem caused by structured and unstructured uncertainties [11]. Wu et al. gave a dissipativity-based sliding mode control method for switched stochastic systems, which has good convergence performance [12]. Li et al. designed a kind of D-stability and no fragile control for discrete-time system based on fuzzy model, which is suitable for descriptor systems with multiple delays [13]. Li et al. proposed a method of stochastic stability for semi-Markovian jump systems, which is found useful in mode-dependent delays [14]. Wu et al. designed an output feedback controller for Markovian jump systems, which provided a new method to control repeated scalar nonlinear systems [15]. The nonlinear part of neural network, uncertainties, and unknown external disturbance were estimated online for the linear system; the equivalent control was realized based on the neural network, and the chattering was eliminated effectively. Ertugrul and Kaynak proposed a mixed sliding mode control method based on neural network, which used two neural networks to approximate equivalent sliding mode control and the part of switching sliding mode control, without object model, and it effectively eliminated the chattering [16]. Huang et al. designed a sliding mode controller by using the approximation ability of RBF neural network; the switching function was regarded as the input of the network; the controller was completely realized by continuous RBF function, and this method canceled the switching part and eliminated the chattering [17].

This paper is organized as follows. The next content consists of five sections about reaching law selection and controller design. The state equation of spacecraft is described in Section 2, which is decomposed into three-channel subsystem, and the shortcomings of two conventional reaching laws are pointed out and an explanation on these two reaching laws is made. Section 3 gives the reaching condition of mixed variable speed reaching law and analyses the existence, reaching conditions, stability, and dynamic performance. Section 4 designs controllers of three subsystems separately combined with neural network. Section 5 shows that, compared with exponential reaching law and variable speed reaching law, the proposed reaching law has better suppress chattering effect and better dynamic performance. Finally, we conclude this paper in Section 6.

\section{Spacecraft Motion Model and Problem Preliminary}

2.1. Spacecraft Motion Model. For a spacecraft tracking system, it can be decomposed into three-channel subsystems called pitch channel subsystem, yaw channel subsystem, and roll channel subsystem, respectively.

Consider the spacecraft tracking system as a discretetime uncertain system, and take state vectors which are timevarying parameters as $x_{1 z}=\mathcal{\vartheta}, x_{2 z}=\dot{\vartheta}, x_{1 y}=\psi, x_{2 y}=\dot{\psi}$, $x_{1 x}=\gamma$, and $x_{2 x}=\dot{\gamma}$; the state space equations of pitch channel subsystem, yaw channel subsystem, and roll channel subsystem can be expressed as follows:

$$
\begin{aligned}
{\left[\begin{array}{l}
x_{1 z}(k+1) \\
x_{2 z}(k+1)
\end{array}\right]=} & {\left[\begin{array}{ll}
1 & T \\
0 & 1
\end{array}\right]\left[\begin{array}{l}
x_{1 z}(k) \\
x_{2 z}(k)
\end{array}\right]+\left[\begin{array}{c}
0 \\
\frac{T \cos \gamma}{J_{z}}
\end{array}\right] u_{z}(k) } \\
& +\left[\begin{array}{c}
0 \\
f_{z}(k)
\end{array}\right], \\
{\left[\begin{array}{l}
x_{1 y}(k+1) \\
x_{2 y}(k+1)
\end{array}\right]=} & {\left[\begin{array}{cc}
1 & T \\
0 & 1
\end{array}\right]\left[\begin{array}{l}
x_{1 y}(k) \\
x_{2 y}(k)
\end{array}\right]+\left[\begin{array}{c}
0 \\
\frac{T \cos \gamma}{\cos \vartheta J_{y}}
\end{array}\right] u_{y}(k) } \\
& +\left[\begin{array}{c}
0 \\
f_{y}(k)
\end{array}\right], \\
{\left[\begin{array}{l}
x_{1 x}(k+1) \\
x_{2 x}(k+1)
\end{array}\right]=} & {\left[\begin{array}{cc}
1 & T \\
0 & 1
\end{array}\right]\left[\begin{array}{l}
x_{1 x}(k) \\
x_{2 x}(k)
\end{array}\right]+\left[\begin{array}{c}
0 \\
\frac{T}{J_{x}}
\end{array}\right] u_{x}(k) } \\
& +\left[\begin{array}{c}
0 \\
f_{x}(k)
\end{array}\right],
\end{aligned}
$$

where the pitch angle $\vartheta$, the yaw angle $\psi$, and the roll angle $\gamma$ are referred to the attitude angles. $f_{x}(k), f_{y}(k)$, and $f_{z}(k)$ are the external torques in the shaft $O x, O y$, and $O z . J_{x}, J_{y}$, and $J_{z}$ are the moments of inertia, $u_{x}(k), u_{y}(k)$, and $u_{z}(k)$ are the external torques on the body frame, and $T$ is the sampling time. The spacecraft attitude tracking system is shown as Figure 1. form:

Systems (1), (2), and (3) can be written in the following

$$
x(k+1)=A x(k)+B u(k)+f(k),
$$

where $x(k) \in R^{n}$ is a state vector, $u(k) \in R$ is the control input, $A \in R^{n \times n}$ is a constant matrix, $B \in R^{n}$ is a constant vector, and $f(k) \in R^{l}$ is external interference, which generally cannot be measured.

2.2. Problem Preliminary. To analyse the tracking problem of discrete system, firstly, we should get the error state equation. Assume that $R(k)$ is the command signal of hope, and $x_{e}(k)=$ $e(k)=R(k)-x(k)$. We can get the discrete error equation of state across the system (4):

$$
x_{e}(k+1)=A_{e} x_{e}(k)+B_{e} u(k)+f_{e}(k),
$$

where $A_{e}=A, f_{e}(k)=-A R(k)+R(k+1)-f(k), B_{e}=$ $-B, R(k)=[r(k) d r(k)]^{T}$, and $d r(k)$ is the change rate of position. $x_{e}(k)=[e(k), d e(k)], e(k)$ is error, and $d e(k)$ is the change rate of error.

Select the switch function

$$
s(k)=C_{e} x_{e}(k),
$$

where $C_{e}$ is the constant matrix needed to design and we should guarantee that $C_{e} B_{e} \neq 0$.

In the sliding mode variable structure control system, movement of the system can be divided into two stages, 


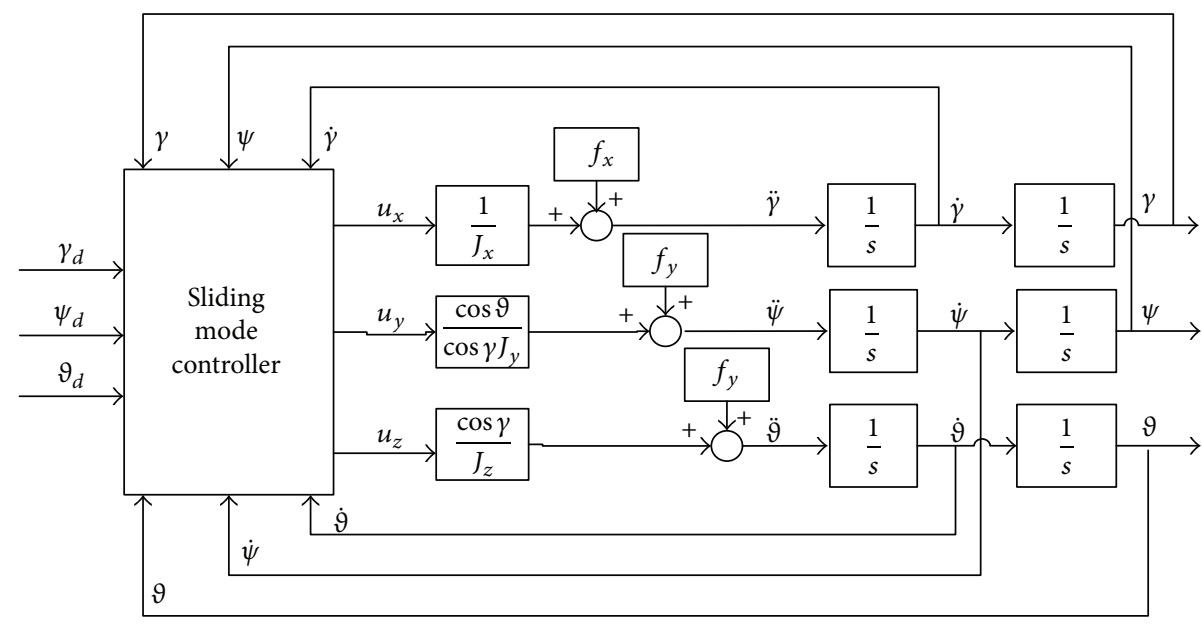

FIGURE 1: The spacecraft attitude tracking system.

respectively, reaching stage and sliding motion stage. The process of system moving to the switching surface from any initial state is called reaching movement, namely, the reaching process of $s \rightarrow 0$ [18]. And the sliding motion is the movement on the surface of sliding mode $s=0$.

Exponential reaching law is a kind of common reaching law for discrete system, and its discrete form is

$$
s(k+1)-s(k)=-q T s(k)-\varepsilon T \operatorname{sgn}(s(k)),
$$

where $\varepsilon>0, q>0,1-q T>0$, and $T$ is the sampling time.

(i) For exponential reaching law, we can get the following formula from (7):

$$
\begin{gathered}
s(k+1)=-\varepsilon T, \quad s(k)=0^{+}, \\
s(k+1)=\varepsilon T, \quad s(k)=0^{-} .
\end{gathered}
$$

Exponential reaching law makes the value of system sliding mode function switch between $\varepsilon T$ and $-\varepsilon T$ during sliding motion stage; namely, its shape of the switching belt is ribbon, which makes the system never reach the origin of coordinate at the end of reaching movement and causes the system chattering.

In order to solve the problem above, variable speed reaching law is considered here [19]. The discrete form of variable speed reaching law is

$$
s(k+1)-s(k)=-\varepsilon T\left\|x_{e}(k)\right\|_{1} \operatorname{sgn}(s(k)),
$$

where $\left\|x_{e}(k)\right\|_{1}=\sum_{i=1}^{n}\left|x_{e i}\right|$ is the system state norm.

(ii) For variable speed reaching law, we can get the following formula from (9):

$$
\begin{array}{cc}
s(k+1)=-\varepsilon T\left\|x_{e}(k)\right\|_{1}, & s(k)=0^{+}, \\
s(k+1)=\varepsilon T\left\|x_{e}(k)\right\|_{1}, & s(k)=0^{-} .
\end{array}
$$

Variable speed reaching law makes the value of system sliding mode function stay in $s(k+1)=0$ during sliding motion stage, because $\left\|x_{e}(k)\right\|_{1}$ will decrease to zero when $s(k)$ approach 0 . We can know that variable speed reaching law uses the entire state vector to control the reaching rate, which can restrain chattering during the sliding motion stage. However, as the value of $\left\|x_{e}(k)\right\|_{1}$ is big when the system enters the switching zone, there exists chattering, which cannot be ignored.

Above all, a mixed variable speed reaching law based on exponential and variable speed reaching law will be studied to eliminate the chattering phenomenon, which has the advantages of two reaching laws.

\section{The Mixed Variable Speed Reaching Law Design and Analysis}

3.1. The Mixed Variable Speed Reaching Law Design. As exponential reaching law is suitable in reaching stage and variable speed reaching law is suitable in sliding motion stage, we can design a kind of reaching law based on these two reaching laws, which can avoid chattering in reaching stage caused by variable speed reaching law and avoid chattering in sliding motion stage caused by exponential reaching law.

The discrete form of the mixed variable speed reaching law is

$$
\begin{aligned}
s(k+1)-s(k)= & \frac{\left\|x_{e}(k)\right\|_{1}+1}{k_{0}}(-q T s(k)-\varepsilon T \operatorname{sgn}(s(k))) \\
& +\frac{k_{0}}{\left\|x_{e}(k)\right\|_{1}+1}\left(-\varepsilon T\left\|x_{e}(k)\right\|_{1} \operatorname{sgn}(s(k))\right),
\end{aligned}
$$

where $k_{0}>0$.

In order to make the system meet the performance requirements, we should analyze the system from the following aspects. 
3.2. Existence and Reaching Condition. Based on the discrete sliding mode theory $[20,21]$, a discrete system should meet the following conditions to guarantee the existence and the reaching condition of sliding mode:

$$
\begin{aligned}
& {[s(k+1)-s(k)] \operatorname{sgn}(s(k))<0,} \\
& {[s(k+1)+s(k)] \operatorname{sgn}(s(k))>0,}
\end{aligned}
$$

where the sampling time $T$ is very small.

Using reaching law (11) to system (5), we can get the function as follows:

$$
\begin{aligned}
& {[s(k+1)-s(k)] \operatorname{sgn}(s(k))} \\
& =\left[\frac{\left\|x_{e}(k)\right\|_{1}+1}{k_{0}}(-q T s(k)-\varepsilon T \operatorname{sgn}(s(k)))\right. \\
& \left.+\frac{k_{0}}{\left\|x_{e}(k)\right\|_{1}+1}\left(-\varepsilon T\left\|x_{e}(k)\right\|_{1} \operatorname{sgn}(s(k))\right)\right] \operatorname{sgn}(s(k)) \\
& =-\frac{\left\|x_{e}(k)\right\|_{1}+1}{k_{0}}(q T|s(k)|+\varepsilon T)-\frac{k_{0} \varepsilon T}{\left\|x_{e}(k)\right\|_{1}+1}\left\|x_{e}(k)\right\|_{1} \\
& <0
\end{aligned}
$$

and when the sampling time $T$ is very small, we can get the following formula:

$$
\begin{aligned}
& {[s(k+1)+s(k)] \operatorname{sgn}(s(k))} \\
& =\left[2 s(k)+\frac{\left\|x_{e}(k)\right\|_{1}+1}{k_{0}}(-q T s(k)-\varepsilon T \operatorname{sgn}(s(k)))\right. \\
& \left.\quad+\frac{k_{0}}{\left\|x_{e}(k)\right\|_{1}+1}\left(-\varepsilon T\left\|x_{e}(k)\right\|_{1} \operatorname{sgn}(s(k))\right)\right] \operatorname{sgn}(s(k)) \\
& =2|s(k)|-T\left[\frac{\left\|x_{e}(k)\right\|_{1}+1}{k_{0}}(q|s(k)|+\varepsilon)\right. \\
& \left.+\frac{k_{0} \varepsilon}{\left\|x_{e}(k)\right\|_{1}+1}\left\|x_{e}(k)\right\|_{1}\right]>0 .
\end{aligned}
$$

Therefore, the mixed variable speed reaching law (11) can satisfy the reaching condition, which can ensure the good dynamic quality for the system.

3.3. Stability Analysis. Assuming $\Delta s(k)=s(k+1)-s(k)$, we can get (15) from (11):

$$
\begin{aligned}
\Delta s(k)= & \frac{\left\|x_{e}(k)\right\|_{1}+1}{k_{0}}(-q T s(k)-\varepsilon T \operatorname{sgn}(s(k))) \\
& +\frac{k_{0}}{\left\|x_{e}(k)\right\|_{1}+1}\left(-\varepsilon T\left\|x_{e}(k)\right\|_{1} \operatorname{sgn}(s(k))\right) .
\end{aligned}
$$

(i) When $s(k)>0$,

$$
\begin{aligned}
\Delta s(k)= & \frac{\left\|x_{e}(k)\right\|_{1}+1}{k_{0}}(-q T s(k)-\varepsilon T) \\
& +\frac{k_{0}}{\left\|x_{e}(k)\right\|_{1}+1}\left(-\varepsilon T\left\|x_{e}(k)\right\|_{1}\right) .
\end{aligned}
$$

From (16), we know that $\Delta s(k)<0$. Therefore, $s(k)$ declines until it is close to the state $s(k)=0$. And only when $s(k)=0$, $\Delta s(k)=0$.

(ii) When $s(k)<0$,

$$
\begin{aligned}
\Delta s(k)= & \frac{\left\|x_{e}(k)\right\|_{1}+1}{k_{0}}(-q T s(k)+\varepsilon T) \\
& +\frac{k_{0}}{\left\|x_{e}(k)\right\|_{1}+1}\left(\varepsilon T\left\|x_{e}(k)\right\|_{1}\right) .
\end{aligned}
$$

From (17), we know that $\Delta s(k)>0$. Therefore, $s(k)$ increases until it is close to the state $s(k)=0$.

(iii) When $s(k)=0$,

$$
s(k+1)=s(k)=0 .
$$

It is easy to known that the system enters into a stable state this moment.

According to the analysis above, in the process of sliding mode motion, the value of $|s(k)|$ is close to 0 infinitely. Thus, the stability of system is fine, and there is no chattering in theory.

3.4. Dynamic Performance Analysis. In the reaching stage, the value of system state norm is bigger as the state vector and does not converge to the switching zone, namely, by selecting $k_{0}$ to satisfy $\left\|x_{e}(k)\right\|_{1}+1 \gg k_{0}$; the unexpected influence caused by variable speed reaching law can be ignored approximately. In sliding motion stage, the state vector is converging in the switching zone, and $\left\|x_{e}(k)\right\|_{1}+$ $1 \ll k_{0}$ can be established, which means that the unexpected influence caused by exponential reaching law is small enough to be ignored at this stage. Therefore, the mixed variable speed reaching law can achieve good dynamic performance in two stages, which combines the advantages of two reaching laws.

In order to analyse the proposed reaching law parameters, the derivation of reaching rate is given here.

If $T$ is small enough, the derivative of continuous system is defined as follows:

$$
\dot{s}(t)=\lim _{\Delta t \rightarrow \infty} \frac{s(t+\Delta t)-s(t)}{\Delta t} .
$$

Therefore, the following equation is available:

$$
\begin{aligned}
\dot{s} & =\frac{s(k+1)-s(k)}{T} \\
& =\frac{\left\|x_{e}\right\|_{1}+1}{k_{0}}(-q s-\varepsilon \operatorname{sgn}(s))+\frac{k_{0}}{\left\|x_{e}\right\|_{1}+1}\left(-\varepsilon\left\|x_{e}\right\|_{1} \operatorname{sgn}(s)\right) .
\end{aligned}
$$


And formula (20) can be written as

$$
\frac{d s}{d t}+q \cdot \frac{\left\|x_{e}\right\|_{1}+1}{k_{0}} s=-\varepsilon \operatorname{sgn}(s)\left[\frac{\left\|x_{e}\right\|_{1}+1}{k_{0}}+\frac{k_{0}\left\|x_{e}\right\|_{1}}{\left\|x_{e}\right\|_{1}+1}\right] .
$$

And, for the first order linear nonhomogeneous differential equation,

$$
\frac{d s}{d t}+P y=Q
$$

Its general solution form [22] is as follows:

$$
s=c e^{-\int P d t}+e^{-\int P d t} \int Q e^{\int P d t} d t .
$$

The constant $c$ is obtained by the initial value $\left.s\right|_{t=0}$.

Take $P=q \cdot\left(\left\|x_{e}\right\|_{1}+1\right) / k_{0}$ and $Q=-\varepsilon \operatorname{sgn}(s)\left[\left(\left(\left\|x_{e}\right\|_{1}+\right.\right.\right.$ $\left.\left.1) / k_{0}\right)+\left(k_{0}\left\|x_{e}\right\|_{1} /\left(\left\|x_{e}\right\|_{1}+1\right)\right)\right]$; we can get the following formula from (23):

$$
\begin{gathered}
s=c e^{-\int q \cdot\left(\left(\left\|x_{e}\right\|_{1}+1\right) / k_{0}\right) d t}+e^{-\int q \cdot\left(\left(\left\|x_{e}\right\|_{1}+1\right) / k_{0}\right) d t} \\
\times \int-\varepsilon \operatorname{sgn}(s)\left[\frac{\left\|x_{e}\right\|_{1}+1}{k_{0}}+\frac{k_{0}\left\|x_{e}\right\|_{1}}{\left\|x_{e}\right\|_{1}+1}\right] \\
\times e^{\int q \cdot\left(\left(\left\|x_{e}\right\|_{1}+1\right) / k_{0}\right) d t} d t .
\end{gathered}
$$

And it can be simplified as

$$
s=c e^{-q \cdot\left(\left(\left\|x_{e}\right\|_{1}+1\right) / k_{0}\right) t}-\frac{\varepsilon}{q} \operatorname{sgn}(s)\left[1+\frac{k_{0}^{2}\left\|x_{e}\right\|_{1}}{\left(\left\|x_{e}\right\|_{1}+1\right)^{2}}\right] .
$$

When $t=0$ and $s=s(0)$,

$$
c=s(0)+\frac{\varepsilon}{q} \operatorname{sgn}(s(0))\left[1+\frac{k_{0}^{2}\left\|x_{e}\right\|_{1}}{\left(\left\|x_{e}\right\|_{1}+1\right)^{2}}\right] .
$$

The relationship between time $t$ and system state $s$ can be obtained by

$$
\begin{aligned}
t & =\frac{k_{0}}{q\left(\left\|x_{e}\right\|_{1}+1\right)} \\
& \cdot \ln \left\{\frac{s(0)+(\varepsilon / q) \operatorname{sgn}(s(0))\left[1+\left(k_{0}^{2}\left\|x_{e}\right\|_{1} /\left(\left\|x_{e}\right\|_{1}+1\right)^{2}\right)\right]}{\left(s+(\varepsilon / q) \operatorname{sgn}(s)\left[1+\left(k_{0}^{2}\left\|x_{e}\right\|_{1} /\left(\left\|x_{e}\right\|_{1}+1\right)^{2}\right)\right]\right)}\right\} .
\end{aligned}
$$

(i) When $s(0)>0$, the time from the initial state $s(0)$ to $s=0^{+}$is

$$
\begin{aligned}
t_{1}= & \frac{k_{0}}{q\left(\left\|x_{e}\right\|_{1}+1\right)} \\
& \cdot \ln \left\{\frac{s(0)+(\varepsilon / q)\left[1+\left(k_{0}^{2}\left\|x_{e}\right\|_{1} /\left(\left\|x_{e}\right\|_{1}+1\right)^{2}\right)\right]}{(\varepsilon / q)\left[1+\left(k_{0}^{2}\left\|x_{e}\right\|_{1} /\left(\left\|x_{e}\right\|_{1}+1\right)^{2}\right)\right]}\right\} \\
= & \frac{k_{0}}{q\left(\left\|x_{e}\right\|_{1}+1\right)} \\
& \cdot \ln \left\{\frac{s(0)}{(\varepsilon / q)\left[1+\left(k_{0}^{2}\left\|x_{e}\right\|_{1} /\left(\left\|x_{e}\right\|_{1}+1\right)^{2}\right)\right]}+1\right\} .
\end{aligned}
$$

And the discrete arrived point for reaching $s(k)=0^{+}$is

$$
\begin{aligned}
k_{1}= & \frac{t_{1}}{T}=\frac{k_{0}}{q T\left(\left\|x_{e}(k)\right\|_{1}+1\right)} \\
& \cdot \ln \left\{\frac{s(0)}{(\varepsilon / q)\left[1+\left(k_{0}^{2}\left\|x_{e}(k)\right\|_{1} /\left(\left\|x_{e}(k)\right\|_{1}+1\right)^{2}\right)\right]}+1\right\} .
\end{aligned}
$$

(ii) When $s(0)<0$, the time from the initial state $s(0)$ to $s=0^{-}$is

$$
\begin{aligned}
t_{2}= & \frac{k_{0}}{q\left(\left\|x_{e}\right\|_{1}+1\right)} \\
& \cdot \ln \left\{\frac{s(0)-(\varepsilon / q)\left[1+\left(k_{0}^{2}\left\|x_{e}\right\|_{1} /\left(\left\|x_{e}\right\|_{1}+1\right)^{2}\right)\right]}{-(\varepsilon / q)\left[1+\left(k_{0}^{2}\left\|x_{e}\right\|_{1} /\left(\left\|x_{e}\right\|_{1}+1\right)^{2}\right)\right]}\right\} \\
= & \frac{k_{0}}{q\left(\left\|x_{e}\right\|_{1}+1\right)} \\
& \cdot \ln \left\{\frac{-s(0)}{(\varepsilon / q)\left[1+\left(k_{0}^{2}\left\|x_{e}\right\|_{1} /\left(\left\|x_{e}\right\|_{1}+1\right)^{2}\right)\right]}+1\right\} .
\end{aligned}
$$

And the discrete arrived point for reaching $s(k)=0^{-}$is

$$
\begin{aligned}
k_{2}= & \frac{t_{2}}{T}=\frac{k_{0}}{q T\left(\left\|x_{e}(k)\right\|_{1}+1\right)} \\
& \cdot \ln \left\{\frac{-s(0)}{(\varepsilon / q)\left[1+\left(k_{0}^{2}\left\|x_{e}(k)\right\|_{1} /\left(\left\|x_{e}(k)\right\|_{1}+1\right)^{2}\right)\right]}+1\right\} .
\end{aligned}
$$

(iii) When $s(0)=0$, we can see that it will be stable at this state $s=s(0)$. 


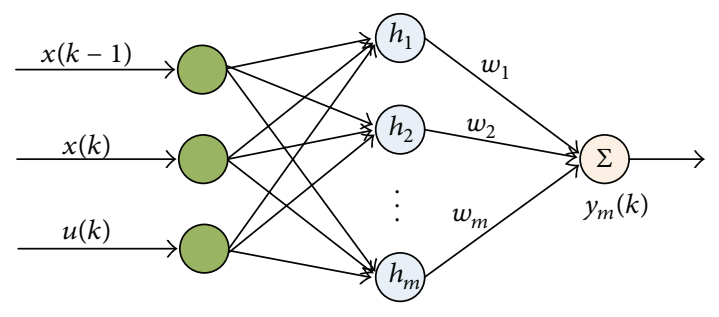

Figure 2: The structure for RBF network.

At this time, in order to accelerate the reaching rate, we should make the discrete point as small as possible. Above all, we can see that parameters $q$, $\varepsilon$, and $k_{0}$ all affect the reaching rate. When $q$ is getting bigger, $\varepsilon$ and $k_{0}$ are getting smaller; the reaching rate is faster, and especially when $q$ is equal to $1 / T$ and $\varepsilon$ is equal to $T$, the reaching rate is the fastest.

\section{Controller Design}

First, we can get the following function for the uncertain system (5):

$$
\begin{aligned}
s(k+1) & =C_{e} x_{e}(k+1) \\
& =C_{e} A_{e} x_{e}(k)+C_{e} B_{e} u(k)+C_{e} f_{e}(k) \\
& =C_{e} R(k+1)-C_{e} A x(k)-C_{e} B u(k)-C_{e} f(k) .
\end{aligned}
$$

From (32), we can get the controller as the following form:

$$
\begin{gathered}
u(k)=\left(C_{e} B\right)^{-1}\left(C_{e} R(k+1)-C_{e} A x(k)-C_{e} f(k)\right. \\
-s(k+1)) .
\end{gathered}
$$

Combined with the proposed mixed variable speed reaching law (11), the controller can be written as

$$
\begin{gathered}
u(k)=\left(C_{e} B\right)^{-1}\left(C_{e} R(k+1)-C_{e} A x(k)-C_{e} f(k)\right. \\
-s(k)-\Delta s(k)),
\end{gathered}
$$

where $\Delta s(k)$ is defined as (15).

In the actual controller, the external disturbance cannot be measured, which may make the controller a big buffeting. Thus, we use a radial basis function neural network (RBF) to estimate the approximation of unknown part, and the RBF network structure is shown in Figure 2, and the RBF is expressed in the form introduced in [23].

This network structure includes three output nodes, $m$ hidden nodes, and one output node. Where $X=$ $\left[x_{1}, x_{2}, x_{3}\right]^{T}=[x(k-1), x(k), u(k)]^{T}$ is the input vector, $\omega_{j}(j=1,2, \ldots, m)$ is written as $w_{j}(k)=w_{j}(k-1)+\eta \Delta w_{j}+$ $\delta\left[w_{j}(k-1)-w_{j}(k-2)\right]$ and $\eta \in(0,1), \delta \in(0,1)$. Assume that $H=\left[h_{1} h_{2} \cdots h_{m}\right]^{T}$ is the radial basis vector set of RBF network, where $h_{j}$ is the Koski function.

4.1. Controller for Pitch Channel Subsystem. Combined with the proposed reaching law and the controller designed for system (5), the controller $u_{z}(k)$ for pitch channel subsystem can be obtained:

$$
\begin{gathered}
u_{z}(k)=\frac{-J_{z}}{c_{2 z} T \cos \gamma}\left[c_{1 z} \vartheta_{d}(k)+c_{2 z} \vartheta_{d}(k+1)-c_{1 z} x_{z 1}(k)\right. \\
-\left(T c_{1 z}+c_{2 z}\right) x_{z 2}(k)-c_{2 z} f_{z}(k) \\
\left.-s_{z}(k)-\Delta s_{z}(k)\right],
\end{gathered}
$$

where $C_{e z}=\left[\begin{array}{ll}c_{1 z} & c_{2 z}\end{array}\right], \vartheta_{d}(k)$ is the command signal for pitch angle, and

$$
\begin{aligned}
\Delta s_{z}(k)= & \frac{\left\|x_{z}(k)\right\|_{1}+1}{k_{0 z}}\left(-q T s_{z}(k)-\varepsilon T \operatorname{sgn}\left(s_{z}(k)\right)\right) \\
& +\frac{k_{0 z}}{\left\|x_{z}(k)\right\|_{1}+1}\left(-\varepsilon T\left\|x_{z}(k)\right\|_{1} \operatorname{sgn}\left(s_{z}(k)\right)\right)
\end{aligned}
$$

is the reaching law of pitch channel subsystem; $k_{0 z}$ is the constant to be designed.

4.2. Controller for Yaw Channel Subsystem. Combined with the proposed reaching law and the controller designed for system (5), the controller $u_{y}(k)$ for yaw channel subsystem can be obtained:

$$
\begin{gathered}
u_{y}(k)=\frac{-c_{2 y} J_{y} \cos \vartheta}{T \cos \gamma}\left[c_{1 y} \psi_{d}(k)+c_{2 y} \psi_{d}(k+1)-c_{1 y} x_{y 1}(k)\right. \\
-\left(T c_{1 y}+c_{2 y}\right) x_{y 2}(k)-c_{2 y} f_{y}(k) \\
\left.-s_{y}(k)-\Delta s_{y}(k)\right],
\end{gathered}
$$

where $C_{e y}=\left[\begin{array}{ll}c_{1 y} & c_{2 y}\end{array}\right], \psi_{d}(k)$ is the command signal for yaw angle, and

$$
\begin{aligned}
\Delta s_{y}(k)= & \frac{\left\|x_{y}(k)\right\|_{1}+1}{k_{0 y}}\left(-q T s_{y}(k)-\varepsilon T \operatorname{sgn}\left(s_{y}(k)\right)\right) \\
& +\frac{k_{0 y}}{\left\|x_{y}(k)\right\|_{1}+1}\left(-\varepsilon T\left\|x_{y}(k)\right\|_{1} \operatorname{sgn}\left(s_{y}(k)\right)\right)
\end{aligned}
$$

is the reaching law of yaw channel subsystem; $k_{0 y}$ is the constant to be designed.

4.3. Controller for Roll Channel Subsystem. Combined with the proposed reaching law and the controller designed for system (5), the controller $u_{x}(k)$ for yaw channel subsystem can be obtained:

$$
\begin{aligned}
u_{x}(k)=\frac{-c_{2 x} J_{x}}{T}[ & c_{1 x} \gamma_{d}(k)+c_{2 x} \gamma_{d}(k+1)-c_{1 x} x_{x 1}(k) \\
& -\left(T c_{1 x}+c_{2 x}\right) x_{x 2}(k)-c_{2 x} f_{x}(k) \\
& \left.-s_{x}(k)-\Delta s_{x}(k)\right]
\end{aligned}
$$



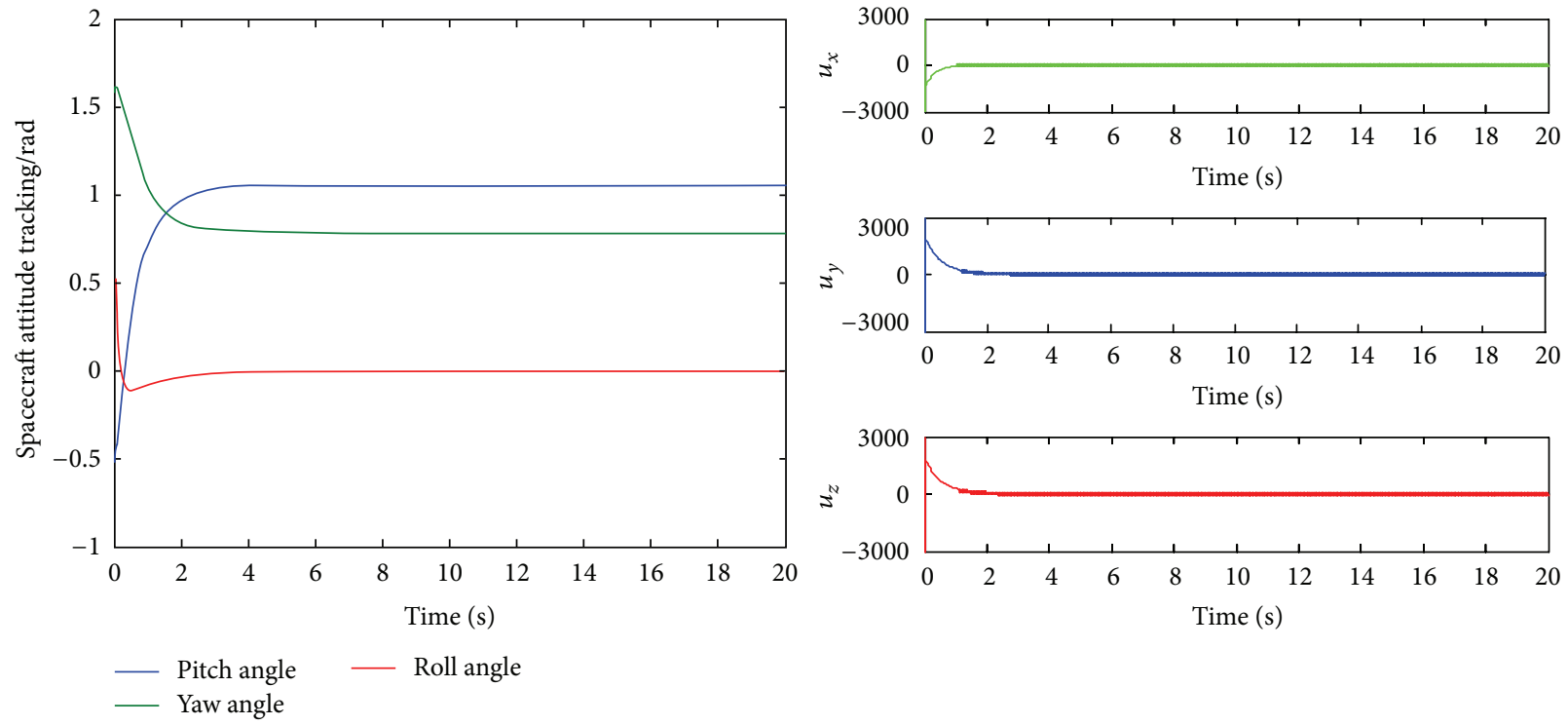

FIGURE 3: Spacecraft attitude tracking and controller output of reaching law (i).
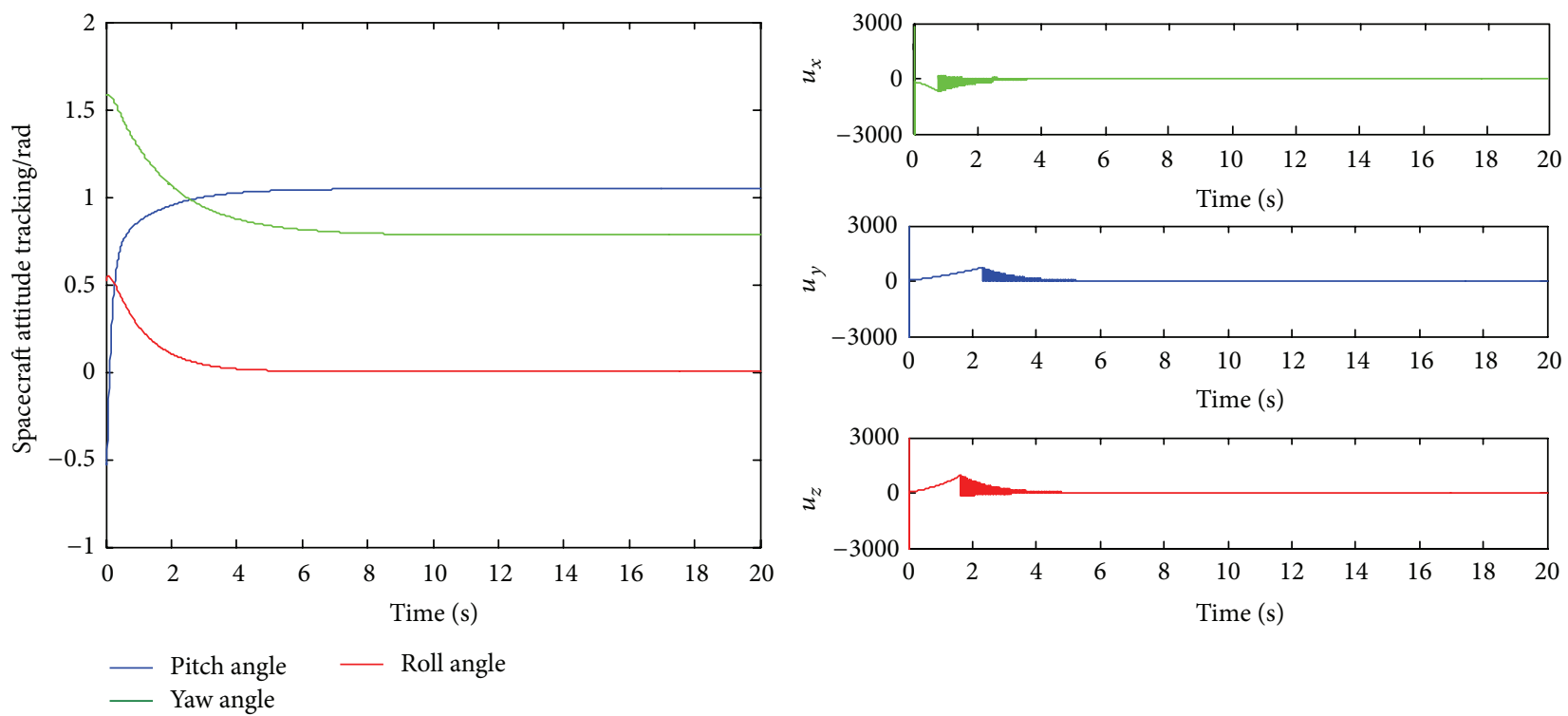

FIGURE 4: Spacecraft attitude tracking and controller output of reaching law (ii).

where $C_{e x}=\left[\begin{array}{ll}c_{1 x} & c_{2 x}\end{array}\right], \gamma_{d}(k)$ is the command signal for roll angle, and

$$
\begin{aligned}
\Delta s_{x}(k)= & \frac{\left\|x_{x}(k)\right\|_{1}+1}{k_{0 x}}\left(-q T s_{x}(k)-\varepsilon T \operatorname{sgn}\left(s_{x}(k)\right)\right) \\
& +\frac{k_{0 x}}{\left\|x_{x}(k)\right\|_{1}+1}\left(-\varepsilon T\left\|x_{x}(k)\right\|_{1} \operatorname{sgn}\left(s_{x}(k)\right)\right)
\end{aligned}
$$

is the reaching law of roll channel subsystem; $k_{0 x}$ is the constant to be designed.

\section{Simulation Study}

In this section, experimental simulations will be carried out to evaluate the effectiveness of the proposed controller based on the mixed variable speed reaching law. The spacecraft motion model in discrete form can be written as formula (41) by putting three subsystems together:

$$
\left[\begin{array}{c}
\dot{x}_{1 z} \\
\dot{x}_{2 z} \\
\dot{x}_{1 y} \\
\dot{x}_{2 y} \\
\dot{x}_{1 x} \\
\dot{x}_{2 x}
\end{array}\right]=\left[\begin{array}{cccccc}
1 & T & 0 & 0 & 0 & 0 \\
0 & 1 & 0 & 0 & 0 & 0 \\
0 & 0 & 1 & T & 0 & 0 \\
0 & 0 & 0 & 1 & 0 & 0 \\
0 & 0 & 0 & 0 & 1 & T \\
0 & 0 & 0 & 0 & 0 & 1
\end{array}\right]\left[\begin{array}{l}
x_{1 z} \\
x_{2 z} \\
x_{1 y} \\
x_{2 y} \\
x_{1 x} \\
x_{2 x}
\end{array}\right]
$$



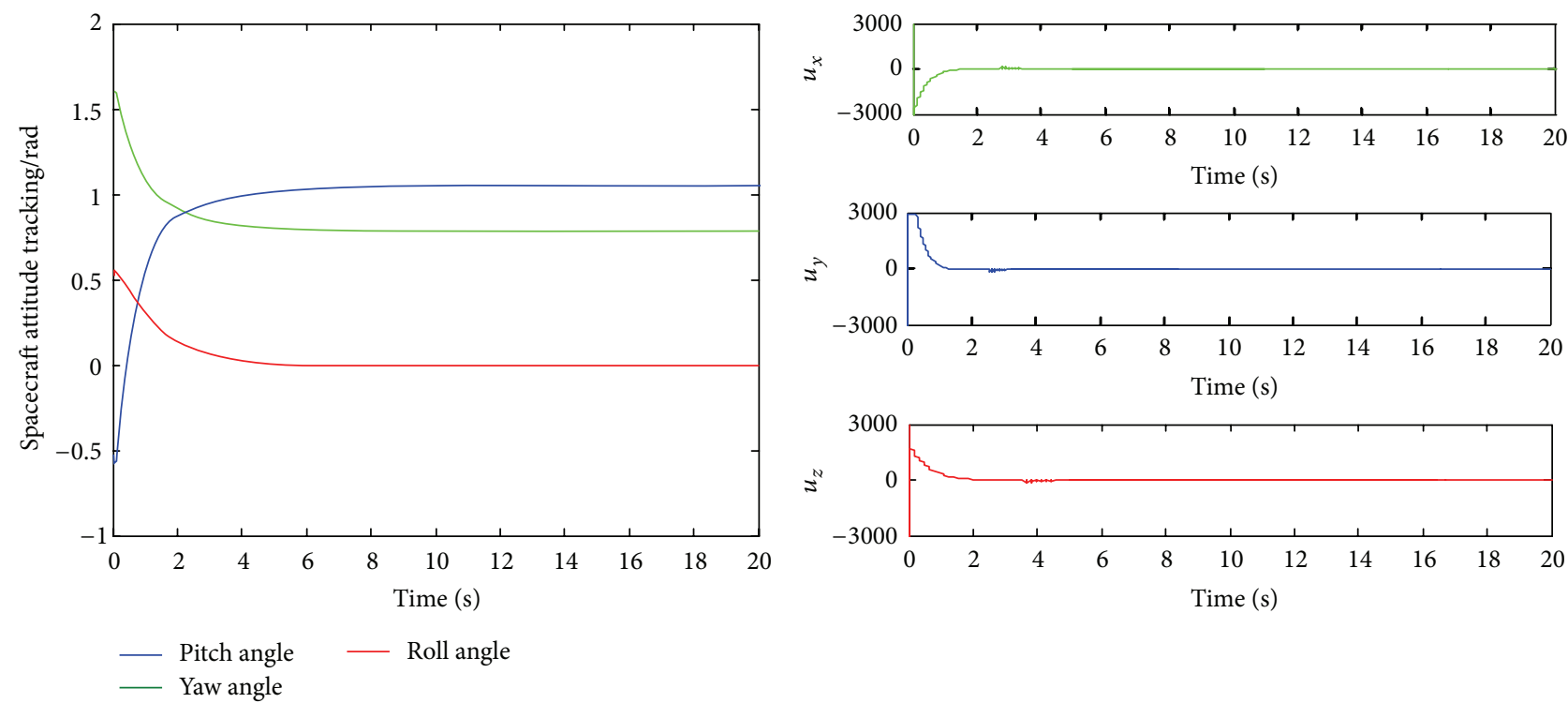

FIGURE 5: Spacecraft attitude tracking and controller output of reaching law (iii).
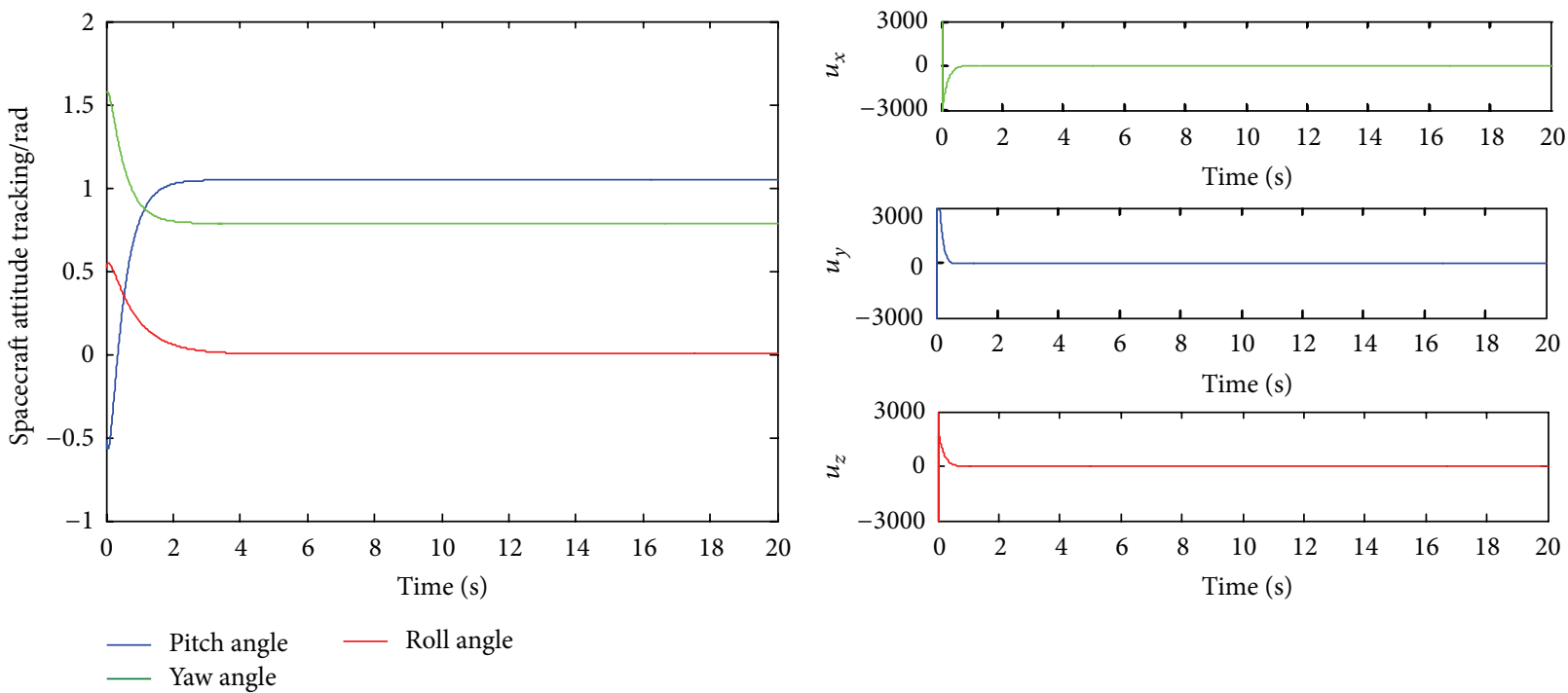

FIGURE 6: Spacecraft attitude tracking and controller output of reaching law (iv).

$$
+\left[\begin{array}{ccc}
0 & 0 & 0 \\
\frac{T \cos x_{1 x}}{J_{z}} & 0 & 0 \\
0 & 0 & 0 \\
0 & \frac{T \cos x_{1 x}}{\cos x_{1 z} J_{y}} & 0 \\
0 & 0 & 0 \\
0 & 0 & \frac{T}{J_{x}}
\end{array}\right]\left[\begin{array}{c}
u_{z} \\
u_{y} \\
u_{x}
\end{array}\right]+\left[\begin{array}{c}
0 \\
f_{z} \\
0 \\
f_{y} \\
0 \\
f_{x}
\end{array}\right] .
$$

Detailed parameters of this control system are presented as follows. We want to stabilize roll angle $\gamma$ to zero, pitch angle $\vartheta$ to a given angle $\vartheta_{d}=\pi / 3$, and yaw angle $\psi$ to a given angle $\psi_{d}=\pi / 4$. Initial condition is $\left[x_{1 z}(0), x_{2 z}(0), x_{1 y}(0), x_{2 y}(0), x_{1 x}(0), x_{2 x}(0)\right]^{T}=$ $[-\pi / 6,0.2, \pi / 2,0.4, \pi / 6,0.5]^{T}$, the system parameters $T=$ $0.02 \mathrm{~s}, J_{x}=224 \mathrm{~kg} \cdot \mathrm{m}^{2}, J_{y}=284 \mathrm{~kg} \cdot \mathrm{m}^{2}$, and $J_{z}=324 \mathrm{~kg} \cdot \mathrm{m}^{2}$. The equivalent interference $f_{z}=f_{y}=f_{x}=\sin (k / 2)$. The controller parameters $C_{e x}=C_{e y}=C_{e z}=\left[\begin{array}{ll}5 & 8\end{array}\right]$, $\left[\begin{array}{lll}k_{o x} & k_{o y} & k_{o z}\end{array}\right]^{T}=\left[\begin{array}{lll}3.06 & 3.30 & 3.15\end{array}\right]^{T}, q=100$, and $\varepsilon=T=$ 0.01 .

Here, we use the following reaching laws to make a comparison with the controlling performance of system.

(i) Exponential reaching law is as follows:

$$
\begin{aligned}
u_{1}(k)=-(C B)^{-1}[ & C A x(k)-(1-q T) s(k) \\
& +\varepsilon T \operatorname{sgn}(s(k))+C f(k)] .
\end{aligned}
$$


(ii) Classical variable speed reaching law is as follows:

$$
\begin{aligned}
u_{2}(k)=-(C B)^{-1}( & C R(k+1)-C A x(k)-s(k) \\
& \left.+\varepsilon T\|x(k)\|_{1} \operatorname{sgn}(s(k))+C f(k)\right) .
\end{aligned}
$$

(iii) Mixed variable speed reaching law is proposed in this paper.

(iv) Mixed variable speed reaching law is proposed in this paper based on neural network.

The spacecraft attitude tracking and controller output of three reaching laws are shown in Figures 3, 4, 5, and 6.

The simulation results show that, in certain interference, the controller outputs of reaching laws (i) and (ii) will produce a severe chattering in the sliding plane. From Figure 3, we know that, under the controller based on exponential reaching law, spacecraft tracking system has chattering phenomenon in sliding motion stage, which cannot decay to zero in a finite time. At the same time, there exists overshoot during attitude tracking process. From Figure 4, it can be seen that, under the controller based on classical variable speed reaching law, spacecraft tracking system has chattering phenomenon in reaching stage, and the chattering amplitude is getting smaller when the norm of state variables convergences to 0 , and there exists no overshoot during attitude tracking process; however, there is a big chattering when the system reaches the switching zone, which cannot be ignored, and the tracking rate becomes slower and slower during attitude tracking process. From Figure 5, it can be seen that, under the controller based on the mixed variable speed reaching law, the spacecraft attitude tracking process is smoothly without overshoot, which has better performance than (i) and (ii). From Figure 6, it is clear that, under the controller based on the mixed variable speed reaching law and neural network, the tracking rate is improved obviously, and system reaches the sliding surface rapidity and achieves the smooth transition with no chattering. Furthermore, the tracking rate is the fastest among four reaching laws and there is no overshoot, which present better dynamic performance.

\section{Conclusions}

A mixed variable speed reaching law of SMC and the controller of three-channel subsystems of spacecraft in the discrete form have been investigated in this paper. It was shown that the proposed reaching law can eliminate chattering completely in theory, and the time of tracking error convergence was significantly shortened compared with exponential reaching law and classical variable speed reaching law; namely, the dynamic character of the system was greatly improved. In a certain external interference, the controller output was ensured to be stable owing to the regulating function of neural network and enhanced the robustness of the system, which has high theoretical value and engineering application value. Furthermore, the further work can be provided in extending the proposed method to timedelay systems and stochastic jump system.

\section{Conflict of Interests}

The authors declare that there is no conflict of interests regarding the publication of this paper.

\section{Acknowledgments}

This work was supported by the National Natural Science Foundation of China (no. 51379049) and the Fundamental Research Funds for the Central Universities of China (nos. HEUCF110419 and HEUCFX41302).

\section{References}

[1] J.-K. Liu and F.-C. Sun, "Research and development on theory and algorithms of sliding mode control," Control Theory \& Applications, vol. 24, no. 3, pp. 407-418, 2007.

[2] J. K. Liu, MATLAB Simulation for Sliding Mode Control, pp. 4-9, Tsinghua University Press, 2005.

[3] W. Gao, Y. Wang, and A. Homaifa, "Discrete-time variable structure control systems," IEEE Transactions on Industrial Electronics, vol. 42, no. 2, pp. 117-122, 1995.

[4] A. J. Koshkouei and A. S. I. Zinober, "Sliding mode control of discrete-time systems," Journal of Dynamic Systems, Measurement and Control, vol. 122, no. 4, pp. 793-802, 2000.

[5] X. Chen, "Adaptive sliding mode control for discrete-time multi-input multi-output systems," Automatica, vol. 42, no. 3, pp. 427-435, 2006.

[6] A. Bartoszewicz, "Discrete-time quasi-sliding-mode control strategies," IEEE Transactions on Industrial Electronics, vol. 45, no. 4, pp. 633-637, 1998.

[7] N. Zaidi, H. B. Azza, M. Jemli, M. Boussak, and A. Chaari, "DSP implementation of speed vector control for single-phase induction motor based on proportional sliding mode control law," International Journal of Innovative Computing, Information and Control, vol. 9, no. 7, pp. 2727-2740, 2013.

[8] Y. Niu, D. W. C. Ho, and Z. Wang, "Improved sliding mode control for discrete-time systems via reaching law," IET Control Theory \& Applications, vol. 4, no. 11, pp. 2245-2251, 2010.

[9] B. Veselic, B. Perunicic-Drazenovic, and C. Milosavljevic, "Improved discrete-time sliding mode position control using Euler velocity estimation," IEEE Transactions on Industrial Electronics, vol. 57, no. 11, pp. 3840-3847, 2010.

[10] L. Wu, X. Su, and P. Shi, "Sliding mode control with bounded $L_{2}$ gain performance of Markovian jump singular time-delay systems," Automatica, vol. 48, no. 8, pp. 1929-1933, 2012.

[11] M. R. Soltanpour, B. Zolfaghari, M. Soltani, and M. H. Khooban, "Fuzzy sliding mode control design for a class of nonlinear systems with structured and unstructured uncertainties," International Journal of Innovative Computing, Information and Control, vol. 9, no. 7, pp. 2713-2726, 2013.

[12] L. Wu, W. X. Zheng, and H. Gao, "Dissipativity-based sliding mode control of switched stochastic systems," IEEE Transactions on Automatic Control, vol. 58, no. 3, pp. 785-791, 2013.

[13] F. Li, P. Shi, and L. Wu, "Fuzzy-model-BasedD-stability and nonfragile control for discrete-time descriptor systems with multiple delays," IEEE Transactions on Fuzzy Systems, vol. 22, no. 4, pp. 1019-1025, 2014.

[14] F. Li, L. Wu, and P. Shi, "Stochastic stability of semi-Markovian jump systems with mode-dependent delays," International Journal of Robust and Nonlinear Control, 2013. 
[15] L. Wu, X. Su, and P. Shi, "Output feedback control of Markovian jump repeated scalar nonlinear systems," IEEE Transactions on Automatic Control, vol. 59, no. 1, pp. 199-204, 2014.

[16] M. Ertugrul and O. Kaynak, "Neuro sliding mode control of robotic manipulators," Mechatronics, vol. 10, no. 1-2, pp. 239$263,2000$.

[17] S.-J. Huang, K.-S. Huang, and K.-C. Chiou, "Development and application of a novel radial basis function sliding mode controller," Mechatronics, vol. 13, no. 4, pp. 313-329, 2003.

[18] F. Li and X. Zhang, "A delay-dependent bounded real lemma for singular LPV systems with time-variant delay," International Journal of Robust and Nonlinear Control, vol. 22, no. 5, pp. 559574, 2012.

[19] K. Furuta, "Sliding mode control of a discrete system," Systems and Control Letters, vol. 14, no. 2, pp. 145-152, 1990.

[20] Y. Niu, D. W. C. Ho, and Z. Wang, "Improved sliding mode control for discrete-time systems via reaching law," IET Control Theory \& Applications, vol. 4, no. 11, pp. 2245-2251, 2010.

[21] C.-C. Gao, Y.-L. Liu, and Y.-Y. Li, "A reaching-law method for uncertain discrete variable-structure control systems," Control Theory and Applications, vol. 26, no. 7, pp. 781-785, 2009.

[22] L. F. Liu, "Application and ordinary solution of A kind of first order differential equation," Journal of Huangshi Institute of Technology, vol. 27, no. 4, pp. 41-42, 2011.

[23] Y. X. Zhao, T. Wu, and Y. Ma, "A double power reaching law of sliding mode control based on neural network," Mathematical Problems in Engineering, vol. 2013, Article ID 408272, 9 pages, 2013. 


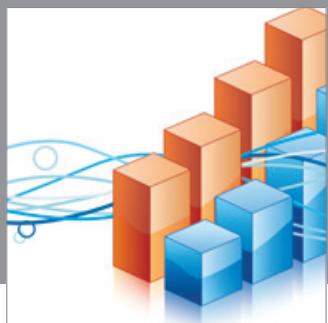

Advances in

Operations Research

mansans

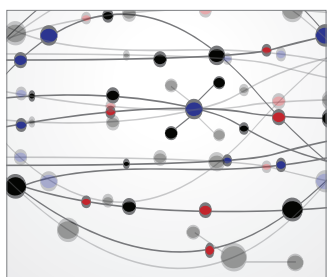

The Scientific World Journal
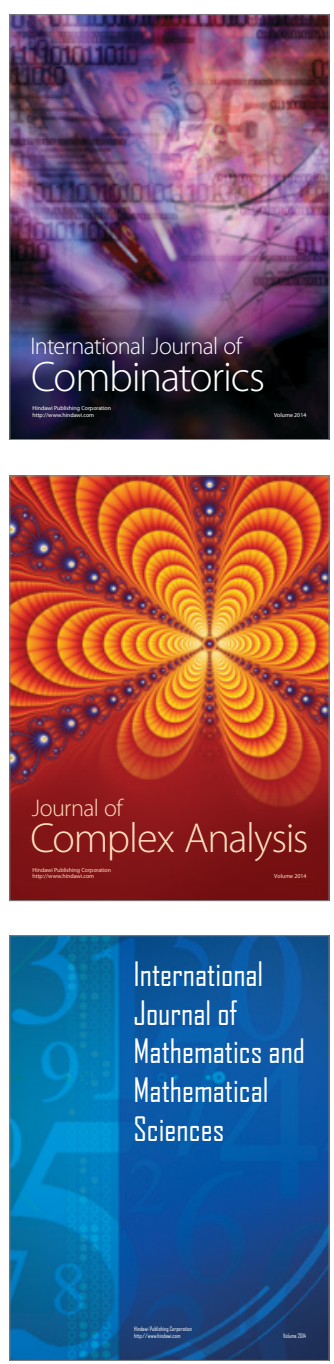
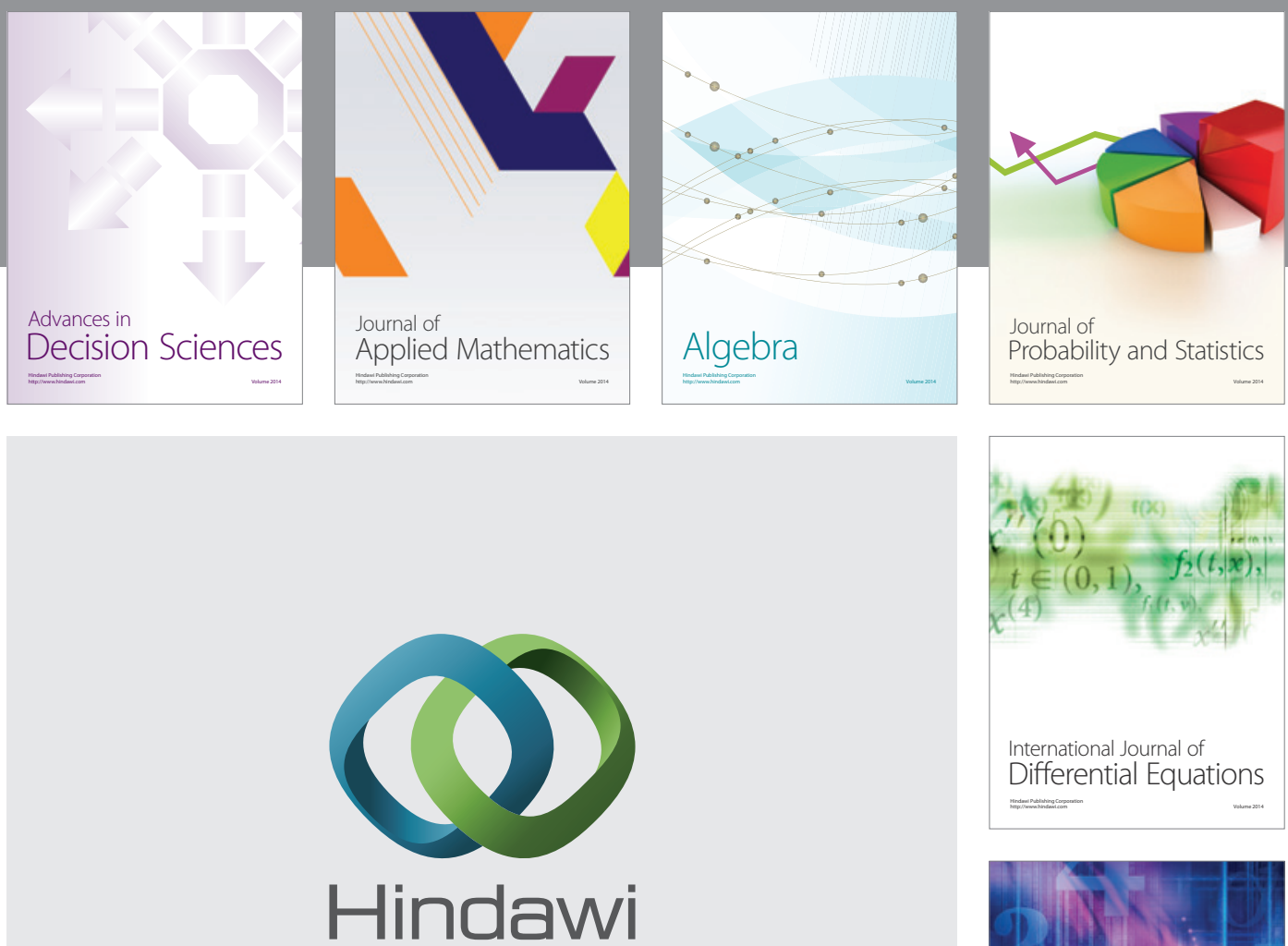

Submit your manuscripts at http://www.hindawi.com
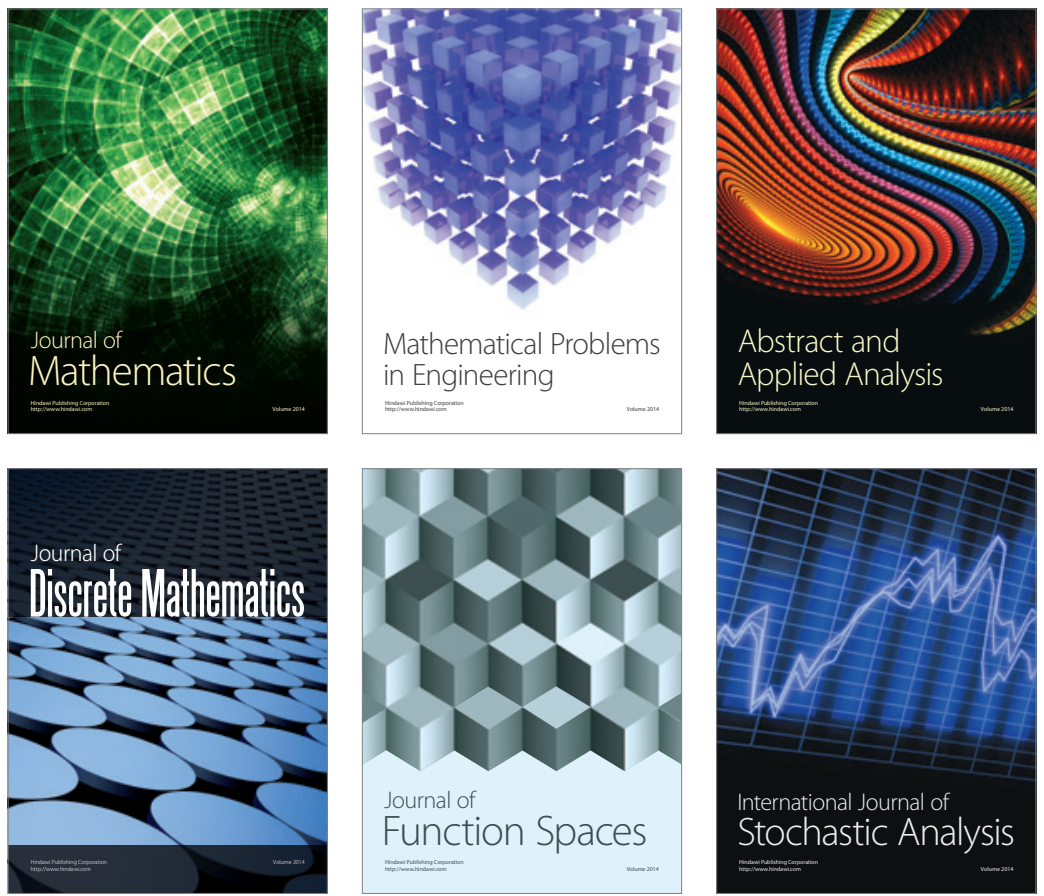

Journal of

Function Spaces

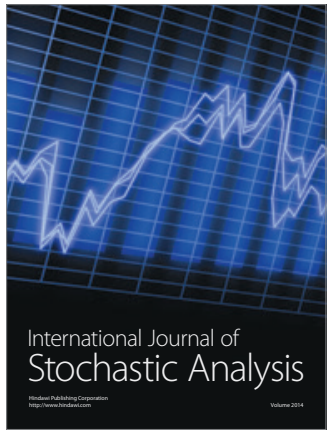

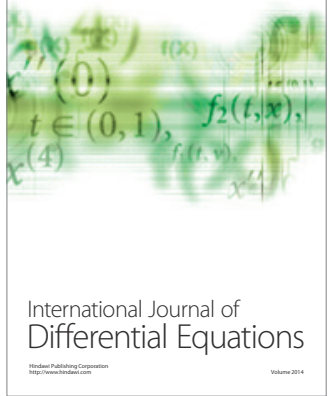
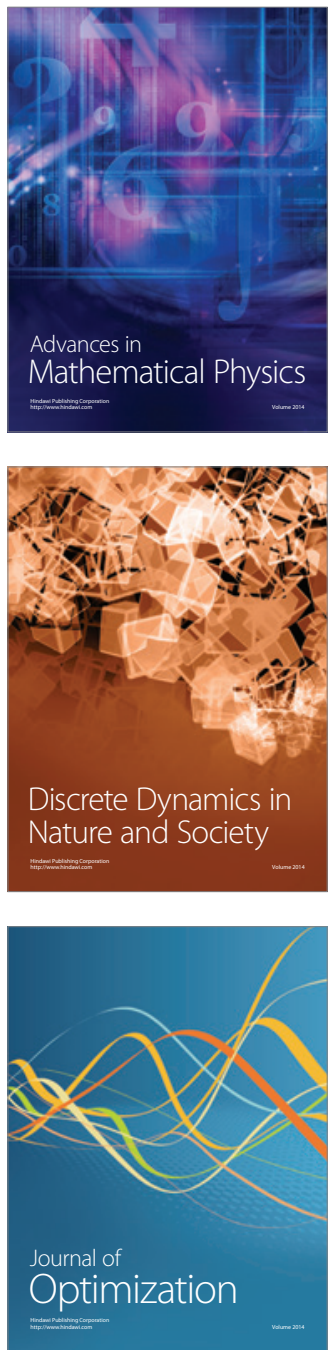